and $76 \%$ were due to neurocysticercosis. (Arya R, Gulati S, Kabra M, Sahu JK, Kalra V. Folic acid supplementation prevents phenytoin-induced gingival overgrowth in children. Neurology April 12, 2011;76:1338-1343). (Response and reprints: Dr Sheffali Gulati, Department of Pediatrics, AIIMS, Ansari Nagar, New Delhi, 110 029, India. E-mail: sheffaligulati@gmail.com).

COMMENT. Gingival hyperplasia associated with phenytoin treatment of epilepsy is reported in as few as $3 \%$ of cases (Lennox WG, 1940) to as many as $78 \%$ (Gardner AF et al, 1962). It occurs more frequently in children than in adults. Numerous mechanisms have been proposed but few of proven significance. Other hydantoin anticonvulsants (mephenytoin, ethotoin, and albutoin) cause little or no gingival hyperplasia. The above investigators have discovered an important and correctable factor in the mechanism in their clinic population. The authors allude to a lack of dental hygiene in a high proportion of patients, a known contributing factor associated with tissue inflammation and irritation. Hyperplasia does not occur in edentulous adults. Phenytoin has an affinity for gingival tissue, and its effect on collagen and keratin in connective tissue has been used in the promotion of healing of wounds and leg ulcers (Shafer WG et al, 1958; Houck JC et al, 1972). In addition to man, only the ferret is susceptible to phenytoin gum hyperplasia, an interesting companion in science. Mechanisms largely disproven include a deficiency of ascorbic acid, adrenocortical dysfunction, and allergy (Gardner, 1962). Hyperglycemia induced by phenytoin, an effect discovered in our neurology research laboratories at Children's Memorial Hospital (Belton NR et al. Epilepsia 1965;6:243-249), warrants investigation as a potential contributing cause of gingival inflammation and overgrowth.

\title{
VALPROIC ACID-ASSOCIATED LIVER FAILURE
}

Researchers at the University of Maryland School of Medicine, Baltimore, and other centers analyzed the records of 17 children undergoing liver transplantation (LT) for valproic acid-associated liver failure (VPA-ALF) and 98 with ALF caused by other drugs (non-VPA-drug-induced acute liver failure [DIALF]. A case report of an 18month-old boy with ALF during treatment with VPA is diagnosed with a mitochondrial disease with 2 distinct heterogeneous missense mutations in DNA POLGI. He was declined as a candidate for LT because of the poor prognosis of LT after VPA-ALF, and he recovered after discontinuation of VPA and initiation of carnitine therapy. In the record review of 17 patients transplanted for VPA-ALF, 14 (82\%) died within 1 year of LT, whereas the 1-year survival for children who underwent LT for non-VPA-DIALF was $69 \%$. $(\mathrm{P}<.0001)$. Except for a lower median alanine aminotransferase level at transplant in VPA-ALF compared with non-VPA-DIALF (45 vs $1179 \mathrm{IU} / \mathrm{L}, \mathrm{P}=.004$ ), pre- and post-transplant parameters of the two groups were comparable. Median post-LT survival time for VPA-ALF was 2.8 months. Children undergoing LT for VPA-ALF have a significantly lower survival probability than children with non-VPA-DIALF. VPA-ALF may represent an unmasking of mitochondrial disease and should be contraindicated for LT, even in absence of mitochondrial disease. In this series, an association with mitochondrial disease was not confirmed. (Mindikoglu AL, King D, Magder LS, Ozolek JA, Mazariegos GV, Shnieder BL. Valproic acid-associated acute liver failure in 
children: case report and analysis of liver transplantation outcomes in the United States. J Pediatr May 2011;158:802-807). (Response and reprints: Ayse L Mindikoglu MD, U Maryland Sch of Med, Baltimore, MD. E-mail: amindiko(amedicine.umaryland.edu).

COMMENT. Children who undergo liver transplantation for VPA-associated acute liver failure have a significantly worse survival rate compared with children with LF caused by other drugs. The authors conclude that in a child with a progressive seizure disorder and acute liver failure after VPA therapy, the VPA should be discontinued and carnitine initiated while investigating a presumed mitochondrial disorder. Liver transplant should be contraindicated for VPA-associated liver failure, even when a mitochondrial disease is not confirmed.

\section{NEURODEGENERATIVE DISORDERS}

\section{CARDIAC INVOLVEMENT IN BATTEN DISEASE}

The onset and progression of cardiac involvement in juvenile neuronal ceroid lipofuscinosis (Batten disease) are studied in 29 children and adolescents with genetically verified disease at Aarhus University Hospital, Skejby, Denmark. One third of initial EKGs had abnormal deeply inverted $T$ waves. Repolarization disturbance of ventricular myocardium at initial recording correlated with risk of death during the 7-year observation period. Heart rate and variability were significantly reduced with increasing age, suggesting a decreased parasympathetic activity on the heart or negative influence on sinus node automaticity. Bradycardia, arrhythmia, sinus arrests and atrial flutter indicated an age-dependent decrease in sinus node activity. In their early 20 s, ventricular hypertrophy was a frequent finding. (Ostergaard JR, Rasmussen TB, Molgaard H. Cardiac involvement in juvenile neuronal ceroid lipofuscinosis (Batten disease). Neurology April 8, 2011;76:1245-1251). (Response and reprints: Dr John R Ostergaard, Department of Pediatrics, Aarthus University Hospital, Skejby, Denmark, E-mail: john.ostergaard@skejby.rm.dk).

COMMENT. Juvenile neuronal ceroid lipofuscinosis (JNCL, Batten disease), caused by mutations in the CLN3 gene and failure to respond to oxidative stress (Tuxworth RJ. Hum Mol Genet 2011; Mar 15[Epub ahead of print]), is the most common type of inherited lysosomal storage and neurodegenerative disease. The clinical course is characterized by progressive visual failure, dementia, and seizures. In JNCL, visual failure occurs at age 4-7 years, and blindness within a few years. Psychomotor deterioration becomes evident in the early school years, and seizures start at a mean age of 10 years. Extrapyramidal symptoms develop at age 12-15, and death usually occurs by the $3^{\text {rd }}$ decade. Cardiac complications have not previously received much attention. In Menkes Textbook of Child Neurology $3^{\text {rd }}$ ed, 1985, cardiac involvement is not mentioned among the various forms of NCL, classified according to age at onset: Infantile (Santavuori) 9-19 months; Late-infantile (Batten-Bielschowsky) 2-4 years; Variant (Batten) 5-7 years; Juvenile (Spielmeyer-Vogt) 4 years-puberty. To-date, 10 types of NCL are described caused by mutations in recessively inherited genes, 8 of which are characterized. 